Beginn der 1970er-Jahre gegen Suchterkrankungen entwickelt, bindet Nalmefene an verschiedene Opioid-Rezeptoren; es wird rasch resorbiert, kaum über Cytochrom P450 metabolisiert und hat mit 13 Stunden eine deutlich längere Plasmahalbwertszeit als Naltrexon.

Inzwischen ist es in drei Phase-III-Studien mit insgesamt etwa 2000 Patienten zur Reduktion der Trinkmenge bei Alkoholabhängigkeit geprüft worden. Vor Beginn der Therapie zählten die Teilnehmer pro Monat zwischen 15 und 20 schwere Trinktage (mehr als $60 \mathrm{~g} / \mathrm{d}$ Alkohol), im Schnitt tranken sie pro Tag etwa $80 \mathrm{~g}$ Alkohol, was dem Gehalt einer Flasche Wein entspricht.

\section{Konsum um die Hälfte reduziert}

Bereits die Bereitschaft, an der Studie teilzunehmen, und ein zusätzlicher Motivationskurs führten zu einer deutlichen Reduktion des Alkoholkonsums in den Placebogruppen. Die Patienten tranken im Schnitt etwa $40 \%$ weniger Alkohol, die Zahl der schweren Trinktage reduzierten sie um etwa die Hälfte. Mit Nalmefene war der Erfolg noch etwas besser: Reduktion der Alkoholmenge um etwa 50\%, Rückgang schwerer Trinktage auf acht pro Monat; der Unterschied war jeweils signifikant. Im ersten Monat ging die Trinkmenge am stärksten zurück, sank anschließend nur noch geringfügig bis etwa zum fünften Monat und blieb dann in etwa konstant. Häufigste Nebenwirkungen waren, wie bei Opioid-Antagonisten üblich, Übelkeit, Schwindel, Schlaflosigkeit und Kopfschmerzen. In der Regel, so Mann, traten diese Symptome aber nur transient auf.

Sollte das Medikament die Zulassung erhalten, könnte dies auch Hausärzten die Scheu nehmen, Patienten erstmals auf ihr Alkoholproblem anzusprechen, sagte der Suchtexperte. Denn dann müsse man nicht mehr gleich mit Abstinenz kommen, was vielleicht viele Betroffene brüskiert. Vielmehr könne man sagen, man hat etwas, das dabei hilft, weniger zu trinken. Das würden viele Patienten wohl eher akzeptieren.

(Thomas Müller)

Vortrag "Abhängigkeit und Sucht" bei der Fortbil-

dungsveranstaltung "Psychiatrie Update“ in Köln, 2012

\title{
Morphintherapie ohne Suchtpotenzial?
}

Bei der Morphintherapie verhindert Dextro-Naloxon die Entstehung einer Sucht, indem es die Ausschüttung von Glückshormonen blockiert.

Forschern aus den USA und Australien könnte der lang gesuchte Durchbruch in der Schmerztherapie gelungen sein. Das Team um Mark Hutchinson und Linda Watkins zeigte erfolgreich im Rattenexperiment, dass Dextro-Naloxon bei einer Morphintherapie die Entstehung einer Abhängigkeit verhindert.

\section{Schmerzlinderung unbeeinflusst}

Die Tiere, die mit Dextro-Naloxon vorbehandelt wurden, zeigten bei der anschließenden Gabe von Morphin keine Anzeichen von Abhängigkeit. Die schmerzlindernde Wirkung des Opiats wurde durch die Kombination nicht be- einträchtigt. Im Gegensatz zu Naloxon, ein Antidot bei Opiatüberdosierung, bindet das Enantiomer Dextro-Naloxon nicht am $\mu$-Rezeptor, sondern am „Tolllike-receptor 4“ (TLR4); diesen tragen im Gehirn die Neuronen des Belohnungssystems. Durch die Blockade mit dem Wirkstoff wird der Entwicklung einer Abhängigkeit vorgebeugt. Denn verglichen mit den Kontrolltieren, die nur Morphin erhielten, schütteten die Ratten, welche zusätzlich Dextro-Naloxon erhielten, viel weniger Dopamin aus. Das „Glückshormon“ Dopamin ist der Neurotransmitter des Belohnungssystems.

Im Rahmen weiterer Tierexperimente sollen nun die Zusammenhänge noch besser verstanden werden, bevor in ca. $18 \mathrm{Mo-}$ naten klinische Studien folgen sollen. (mg)

Hutchinson MRet al, J Neurosci, 2012, 32(33):11187-11200

\section{E-Nikotin pro Herz?}

Die E-Zigarette schadet Herz und Gefäßen weniger als herkömmliche Kippen: In einer griechischen Studie verglichen Forscher die akuten Effekte auf die Herzfunktion bei 20 Rauchern und 22 „Dampfern“ (11 mg/ml Nikotin im Test-Liquid); ehemaligen Zigarettenrauchern. Bei allen Rauchern, und nur bei diesen, traten Störungen der diastolischen Herzfunktion auf. Hämodynamisch schnitt der Glimmstängel auch schlechter ab: Bei Rauchern stieg der Blutdruck um $8 \%$ systolisch, 6\% diastolisch; der Puls um 10\% an. Dampfen erhöhte nur den diastolischen Wert um 4\%.

Ärzte Zeitung online

\section{Kombi erleichtert Kokain-Entzug}

Buprenorphin kombiniert mit Naltrexon könnte den Drogenentzug Kokainabhängiger erleichtern. Bisher nur im Tierversuch getestet, zeigte die Kombi bei Ratten die gewünschte Wirkung: Die Tiere verloren dauerhaft das Interesse an der Droge. Buprenorphin, eingesetzt in der HeroinSubstitutionstherapie, verhindert über ähnliche Mechanismen auch bei Kokain Rückfälle. Die Kombination mit Naltrexon verhindert die Opiat-Abhängigkeit.

Wee Set al, Sci Trans Med 2012, 4:146ra110

\section{Steuer gegen Zucker-Sucht}

Zucker ist ähnlich gesundheitsschädigend und suchtfördernd wie Alkohol und Tabak, betonen Ärzte aus Kalifornien. Der Konsum sollte somit auch ähnlich kontrolliert werden. In den letzten 50 Jahren hat sich der Verzehr verdreifacht. Sie fordern deshalb eine Steuer auf Süßes und eine begrenzte Abgabe an Kinder. Auch die British Heart Foundation wünscht sich die Besteuerung ungesunder Lebensmittel. Unterstützend fordern sie die Subventionierung von Obst und Gemüse.

Lustig R Het al, Nature 2012, 482: 27-29 